\title{
SECOND ORDER SUBEXPONENTIAL DISTRIBUTIONS
}

\author{
J. L. GELUK and A. G. PAKES \\ (Received 17 March 1989; revised 6 April 1990) \\ Communicated by Timothy C. Brown
}

\begin{abstract}
The class of subexponential distributions $S$ is characterized by $F(0)=0,1-F^{(2)}(x) \sim$ $2(1-F(x))$ as $x \rightarrow \infty$. In this paper we consider a subclass of $S$ for which the relation $1-F^{(2)}(x)-2(1-F(x))+(1-F(x))^{2}=o(\alpha(x))$ as $x \rightarrow \infty$ holds, where $\alpha$ is a positive function satisfying $\alpha(X)=o(1-F(x)) \quad(x \rightarrow \infty)$.

1980 Mathematics subject classification (Amer. Math. Soc.) (1985 Revision): 60 E 05.
\end{abstract}

\section{Introduction}

In this paper we assume that $F$ is a distribution function on $[0, \infty)$ for which $F(0-)=0, F(x)<1$ for all $x>0$ and $F(\infty)=1$.

The distribution function $F$ is said to belong to the subexponential class (notation: $F \in \mathscr{S}$ ) if

$$
\lim _{x \rightarrow \infty} \overline{F^{(2)}}(x) / \bar{F}(x)=2,
$$

where $\bar{F}(x):=1-F(x)$ and $\overline{F^{(2)}}(x):=1-F^{(2)}(x)$ is the tail of the distribution function of the convolution of $F$ with itself.

In spite of the simple definition of $\mathscr{S}$ there exists no characterization of $\mathscr{S}$ without a convolution. We list a few properties of $\mathscr{S}$.

(i) $\mathscr{S} \subset \mathscr{L}$ where the class $\mathscr{L}$ consists of those distribution functions $F$ satisfying $\bar{F}(x-u) / \bar{F}(x) \rightarrow 1$ as $x \rightarrow \infty$ for $u \in \mathbb{R}$ (uniformly on compact sets).

(C) 1991 Australian Mathematical Society 0263-6115/91 \$A2.00+0.00 
(ii) If $F \in \mathscr{S}$ and $\varepsilon>0$, then $e^{e x} \bar{F}(x) \rightarrow \infty(x \rightarrow \infty)$.

(iii) If $F \in \mathscr{S}$, then $\overline{F^{(k)}}(x) / \bar{F}(x) \rightarrow k \quad(x \rightarrow \infty)$ for all $k \geq 2$.

(iv) If $F \in \mathscr{S}$ and $\bar{G}(x) \sim \bar{F}(x)(x \rightarrow \infty)$, then $G \in \mathscr{S}$.

(v) If $F \in \mathscr{L}$ and $\bar{F}$ is $O$-regularly varying, that is, if

$$
\varlimsup_{x \rightarrow \infty} \bar{F}(a x) / \bar{F}(x)<\infty \text { for } a>0,
$$

then $F \in \mathscr{S}$.

The first three results were proved by Chistyakov [3]; (iv) is proved in Teugels [15] and other authors independently (see Pakes [13]). Sufficient conditions for $F \in \mathscr{S}$ are given in Chistyakov [3], Teugels [15], Pitman [14] and Cline [4]. Pitman's necessary and sufficient condition is formulated in terms of the function

$$
\psi(x):=-\log \bar{F}(x) .
$$

If $\psi$ has a derivative $\psi^{\prime}$ which is eventually non-increasing to 0 , then a necessary and sufficient condition for $F \in \mathscr{S}$ is

$$
\lim _{x \rightarrow \infty} \int_{0}^{x} \exp \left\{y \psi^{\prime}(x)-\psi(y)\right\} \psi^{\prime}(y) d y=1
$$

and a sufficient condition is $\exp \left\{y \psi^{\prime}(y)-\psi(y)\right\} \psi^{\prime}(y)$ is integrable over $(0, \infty)$.

A recent sufficient condition which includes ( $v$ ) and Pitman's sufficient condition is due to Murphree [11].

Suppose $F \in \mathscr{L}$ and there exists a constant $c$ such that

$$
\psi(x)-\psi(u) \leq(x-u) \psi(x) / x+c \text { for all } u \in[x / 2, x]
$$

and

$$
\int_{0}^{\infty} \exp \left\{\frac{1}{2}(\psi(2 x)-2 \psi(x))\right\} d \psi(x)<\infty
$$

Then $F \in \mathscr{S}$.

An analogue of property $(v)$ for densities is well-known. See, for example, Luxemburg [10]. For densities, extensions to second order results are given by Geluk [7], Willekens [16] and Omey [12].

It is well-known, see Chistyakov [3], that if $\bar{F}(x)>0 \quad(x>0)$ then

$$
\varliminf_{x \rightarrow \infty}\left\{\overline{F^{(2)}}(x)-2 \bar{F}(x)\right\} / \bar{F}(x) \geq 0 .
$$

A second order analogue is defined in Lemma 1 below: if $\bar{F}(x)>0$ for $x>0$, then

$$
\varliminf_{x \rightarrow \infty}\left\{\overline{F^{(2)}}(x)-2 \bar{F}(x)+\bar{F}(x)^{2}\right\} / \bar{F}(x)^{2} \geq 0
$$


The class of subexponential distributions $\mathscr{S}$ is given by those d.f.'s $F$ for which the limit in (1) exists and equals 0 . Here we study the case for which the limit in (2) exists and equals 0 . We will denote this class by $\mathscr{S}^{2}$. More generally we consider the class of d.f.'s $F$ for which there exists a positive function $\alpha=o(\bar{F})$ such that

$$
\lim _{x \rightarrow \infty}\left\{\overline{F^{(2)}}(x)-2 \bar{F}(x)+\bar{F}(x)^{2}\right\} / \alpha(x)=0 .
$$

We will denote this class by $\mathscr{S}^{2}(\alpha)$. Observe that $\mathscr{S}^{2}(\alpha) \subset \mathscr{S}$ since the assumption $\alpha=o(\bar{F})$ ensures that (3) is stronger than the usual limit relation defining the class $\mathscr{S}$. Note that the inequality (2) does not say anything about the growth of the function $\alpha$ in (3). We will show by examples that $\bar{F}(x)^{2}=o(\alpha(x)), \alpha(x)=o\left(\bar{F}(x)^{2}\right)$ and $\alpha(x)=\bar{F}(x)^{2}$ are possible in (3). Note that if $F \in \mathscr{S}^{2}(\alpha)$ with $\underline{\lim }_{x \rightarrow \infty} \bar{F}(x)^{2} / \alpha(x)>0$, then it follows that $F \in \mathscr{S}^{2}$. For the class $\mathscr{S}$ the defining relation is equivalent to

$$
\int_{0-}^{x} \frac{\bar{F}(x-y)-\bar{F}(x)}{\bar{F}(x)} d F(y)=o(1) \quad(x \rightarrow \infty) .
$$

The class $\mathscr{S}^{2}(\alpha)$ is characterized by the rate of convergence in the above relation. It is easy to see that the defining relation (3) is equivalent to

$$
\int_{0-}^{x} \frac{\bar{F}(x-y)-\bar{F}(x)}{\bar{F}(x)} d F(y)=o\left(\frac{\alpha(x)}{\bar{F}(x)}\right) \quad(x \rightarrow \infty) .
$$

This explains why the assumption $\alpha=o(\bar{F})$ is natural in the definition of $\mathscr{S}^{2}(\alpha)$.

The proofs of our results are extensions of existing proofs for the class $S$. For the proof of Theorem 4, compare with Chistyakov [3]. Theorem 5 below is an extension to the class $\mathscr{S}^{2}$ of Murphree's [11] result. For applications of the class of subexponential distribution functions the reader is referred to Athreya and Ney [1] and Embrechts [6].

\section{Results}

The first result provides us with a sufficient condition in order to obtain a distributin function $F$ which belongs to $\mathscr{S}^{2}(\alpha)$.

THEOREM 1. If there exists a positive function $\alpha=o(\bar{F})$ such that

$$
\lim _{x \rightarrow \infty} \frac{\bar{F}(x-u)-\bar{F}(x)}{\alpha(x)}=0 \text { for } u>0
$$


and

$$
\varlimsup_{x \rightarrow \infty} \frac{\bar{F}(x / 2)-\bar{F}(x)}{\alpha(x)}<\infty,
$$

then $F \in \mathscr{S}^{2}(\alpha)$. In particular, if (4) and (5) are satisfied with $\alpha(x)=\bar{F}(x)^{2}$, then $F \in \mathscr{S}^{2}$.

Proof. Observe that

$\overline{F^{(2)}}(x)-2 \bar{F}(x)+\bar{F}(x)^{2}=2 \int_{0-}^{x / 2}(\bar{F}(x-u)-\bar{F}(x)) d F(u)+(\bar{F}(x / 2)-\bar{F}(x))^{2}$.

In view of our assumptions we may divide by $\alpha(x)$ and then apply Lebesgue's dominated convergence theorem.

REMARK. If we drop the assumption $\alpha=o(\bar{F})$, then (3) follows and the above theorem is a generalization of property $(v)$ in the introduction (take $\alpha(x)=\bar{F}(x))$.

The following corollary gives a link with the theory of slow variation with remainder. See, for example, Goldie and Smith [9].

Corollary 1. (a) If $1-\bar{F}(x) / \bar{F}(x / 2)=O(\alpha(x))(x \rightarrow \infty)$ with a positive function $\alpha$ satisfying $\alpha=o(\bar{F})$, then $F \in \mathscr{S}^{2}(\alpha)$.

(b) If $1-\bar{F}(x) / \bar{F}(x / 2)=o(\bar{F}(x))(x \rightarrow \infty)$, then $F \in \mathscr{S}^{2}$.

(c) If there exists a positive function $\alpha$ such that

$$
\frac{\bar{F}(t x)-\bar{F}(x)}{\alpha(x)} \rightarrow-\log t \quad(x \rightarrow \infty) \text { for } t>0,
$$

then $F \in \mathscr{S}^{2}(\alpha)$.

Proof. Under assumption (a), (5) follows directly and (4) follows since

$$
0 \leq \frac{\bar{F}(x-u)-\bar{F}(x)}{\alpha(x)} \leq\left(1-\frac{\bar{F}(x)}{\bar{F}(x / 2)}\right) \frac{\bar{F}(x / 2)}{\alpha(x)} \rightarrow 0 \quad(x \rightarrow \infty) .
$$

Note that assumption (b) implies (4) and (5) with $\alpha(x)=\bar{F}(x)^{2}$.

Convergence in the limit under (c) is uniform for $t \in[a, b]$ with $0<$ $a<b<\infty$ (due to monotonicity of $\bar{F}$ ). This implies (4). Note that (5) is satisfied by assumption and $\alpha=o(\bar{F})$ by Remark 1 on page 25 in [8]. (For further properties of functions satisfying (c) see also [2, Chapter 3].)

ReMARK. If for $\delta>0 \bar{F}(x)=(\log x)^{-\delta}+o(\log x)^{-\delta-1}(x \rightarrow \infty)$, then $F$ satisfies (c) above with $\alpha(x)=\delta(\log x)^{-\delta-1}$. It follows that for $\delta=1$, $\alpha(x) \sim \bar{F}(x)^{2}$, and hence $F \in \mathscr{S}^{2}$. If $0<\delta<1$, then $\alpha(x)=o\left(\bar{F}(x)^{2}\right)$ which together with (3) implies $F \in \mathscr{S}^{2}$. If $\delta>1$, then $\bar{F}(x)^{2}=o(\alpha(x))$, and hence $\overline{F^{(2)}}(x)-2 \bar{F}(x)=o(\log x)^{-\delta-1}$. 
Question: is $F \in \mathscr{S}^{2}$ when $\delta>1$ ? This question is not answered; however we prove that $F \in \mathscr{S}^{2}$ if $\bar{F}(x)=(\log x)^{-\delta}+o(\log x)^{-2 \delta} \quad(x \rightarrow \infty)$. See Remark 1 after Theorem 6.

Next we will give analogues of the results (i) to (iv) mentioned in the introduction. First we give an analogue of (i).

THEOREM 2. If $F \in \mathscr{S}^{2}(\alpha)$ then

$$
\bar{F}(x-A)-\bar{F}(x)=o(\alpha(x)) \quad(x \rightarrow \infty) \text { for any fixed } A>0
$$

and

$$
\begin{aligned}
&\{\bar{F}(x-A)-\bar{F}(x)\}\{\bar{F}(A)-\bar{F}(x)\}=o(\alpha(x)) \\
&(x \rightarrow \infty) \text { for any } A=A(x) \in(0, x) .
\end{aligned}
$$

Proof. Observe that since

$$
\overline{F^{(2)}}(x)-2 \bar{F}(x)+\bar{F}(x)^{2}=\int_{0-}^{x}\{\bar{F}(x-u)-\bar{F}(x)\} d F(u)
$$

it follows that $0<\{\bar{F}(x-A)-\bar{F}(x)\}\{\bar{F}(A)-\bar{F}(x)\} \leq \overline{F^{(2)}}(x)-2 \bar{F}(x)+\bar{F}(x)^{2}$ for $A=A(x) \in(0, x)$ and $x>0$. This implies (7). Moreover (6) follows if we take $A>0$ constant.

Corollary 2. If $F \in \mathscr{S}^{2}(\alpha)$ with $\alpha(x)=O\left(\bar{F}(x)^{2}\right) \quad(x \rightarrow \infty)$, then $\bar{F}$ is $O$-regularly varying. If $F \in \mathscr{S}^{2}$ then $\bar{F}$ is slowly varying and satisfies

$$
\bar{F}(x-A)-\bar{F}(x)=o\left(\bar{F}(x)^{2}\right) \quad(x \rightarrow \infty) \text { for } A>0,
$$

or, equivalently, $\bar{F}(x)^{-1}-\bar{F}(x-A)^{-1} \rightarrow 0(x \rightarrow \infty)$ for $A>0$.

REMARK. It should be observed that d.f.'s with a RO varying tail and even d.f.'s with a regularly varying tail generally do not belong to $\mathscr{S}^{2}(\alpha)$. For example, if $F$ is a one-sided stable distribution of index $1 / 2$, then $\bar{F}(x) \sim c_{1} x^{-1 / 2} \quad(x \rightarrow \infty)$; however $\bar{F}^{(2)}(x)-2 \bar{F}(x) \sim c_{2} \bar{F}(x)^{3} \quad(x \rightarrow \infty)$ for some constants $c_{1}, c_{2}$, and hence $F \notin \mathscr{S}^{2}(\alpha)$.

Next we will prove a closure property related to the class $\mathscr{S}^{2}(\alpha)$ which can be seen as an analogue of property (iv).

THEOREM 3. Suppose

$$
F \in \mathscr{S}^{2}(\alpha)
$$

and

$$
\bar{F}(x)^{2} / \alpha(x) \rightarrow d \quad(x \rightarrow \infty) \text { with } 0 \leq d<\infty .
$$


If

$$
\bar{G}(x)=K \bar{F}(x)+(c+o(1)) \bar{F}(x)^{2} \quad(x \rightarrow \infty)
$$

with $K>0, c \in \mathbb{R}$, then

$$
\left\{\overline{G^{(2)}}(x)-2 \bar{G}(x)\right\} / \alpha(x) \rightarrow-K^{2} d
$$

whence $G \in \mathscr{S}^{2}(\alpha)$.

Proof. Since $\overline{G^{(2)}}(x)-2 \bar{G}(x)=\int_{0-}^{x}\{\bar{G}(x-y)-\bar{G}(x)\} d G(y)-\bar{G}(x)^{2}$, it follows that we have to show

$$
\int_{0-}^{x}\{\bar{G}(x-y)-\bar{G}(x)\} d G(y)=o(\alpha(x)) \quad(x \rightarrow \infty) .
$$

Given $\varepsilon>0$ there exists $a>0$ such that

$$
\bar{G}(x)=K \bar{F}(x)+(c+r(x)) \bar{F}(x)^{2} \quad(x>0)
$$

and $|r(x)|<\varepsilon$ for $x>a$. Consequently the integral in (13) is

$$
\begin{aligned}
K \int_{0-}^{x-a} & \{\bar{F}(x-y)-\bar{F}(x)\} d G(y)+c \int_{0-}^{x-a}\left\{\bar{F}(x-y)^{2}-\bar{F}(x)^{2}\right\} d G(y) \\
& +\int_{0-}^{x-a} r(x-y) \bar{F}(x-y)^{2} d G(y)+o\left(\bar{F}(x)^{2}\right) \\
& +\int_{x-a}^{x}\{\bar{G}(x-y)-\bar{G}(x)\} d G(y) \\
= & : \mathrm{I}_{1}+\mathrm{I}_{2}+\mathrm{I}_{3}+o\left(\bar{F}^{2}\right)+\mathrm{I}_{4} .
\end{aligned}
$$

Since $F \in \mathscr{S}^{2}(\alpha)$, using (6) it follows that

$$
\begin{aligned}
\mathrm{I}_{4} & \leq G(x)-G(x-a)=\bar{G}(x-a)-\bar{G}(x) \\
& =K\{\bar{F}(x-a)-\bar{F}(x)\}+c\left(\bar{F}(x-a)^{2}-\bar{F}(x)^{2}\right)+o\left(\bar{F}(x)^{2}\right) \\
& =o(\alpha(x))+o\left(\bar{F}(x)^{2}\right)=o(\alpha(x)) \quad(x \rightarrow \infty) .
\end{aligned}
$$

\section{Moreover}

$\mathrm{I}_{3} \leq \int_{0-}^{x-a} r(x-y)\left\{\bar{F}(x-y)^{2}-\bar{F}(x)^{2}\right\} d G(y)+\bar{F}(x)^{2} \int_{0-}^{x-a} r(x-y) d G(y)$.

From dominated convergence it follows that the last term is $o\left(\bar{F}(x)^{2}\right)$. The first term can be dominated by

$$
\begin{aligned}
2 \varepsilon \int_{0-}^{x-a}\{\bar{F}(x-y)-\bar{F}(x)\} d G(y) & \leq 2 \varepsilon \int_{0-}^{x}\{\bar{F}(x-y)-\bar{F}(x)\} d G(y) \\
& =2 \varepsilon \int_{0-}^{x}\{\bar{G}(x-y)-\bar{G}(x)\} d F(y)
\end{aligned}
$$


Note that $\int_{0-}^{x}\{\bar{F}(x-y)-\bar{F}(x)\} d F(y)=\overline{F^{(2)}}(x)-2 \bar{F}(x)+\bar{F}(x)^{2}=o(\alpha(x))$ by (9) from which it follows that if we substitute the expression (14) for $\bar{G}$ in the right-hand side of (15) the result will be $o(\alpha(x))$.

Note that $\mathrm{I}_{2} \leq 2 c \int_{0-}^{x}\{\bar{F}(x-y)-\bar{F}(x)\} d G(y)$ which can be handled as the integral in (15) and the same is true for $I_{1}$.

In the sequel we need the following lemma, which improves [3, Lemma 1].

LEMMA 1.

$$
\begin{aligned}
-\left(\begin{array}{l}
n \\
2
\end{array}\right) & \leq \varliminf_{x \rightarrow \infty} \frac{\overline{F^{(n)}}(x)-n \bar{F}(x)}{\bar{F}(x)^{2}} \leq \varlimsup_{x \rightarrow \infty} \frac{\overline{F^{(n)}}(x)-n \bar{F}(x)}{\bar{F}(x)^{2}} \\
& \leq \varlimsup_{x \rightarrow \infty} \frac{1-F\left(\frac{x}{n}\right)^{n}-n \bar{F}(x)}{\bar{F}(x)^{2}} .
\end{aligned}
$$

We omit the proof of this lemma which is based on the same inequalities as those used in the proof of [3, Lemma 1].

THEOREM 4. If $F \in \mathscr{S}^{2}$, then

$$
\lim _{x \rightarrow \infty} \frac{\overline{F^{(n)}}(x)-n \bar{F}(x)}{\bar{F}(x)^{2}}=-\left(\begin{array}{l}
n \\
2
\end{array}\right) \text { for } n \geq 2 .
$$

Proof. The proof is by induction. For $n=2$ the statement of the theorem is correct. Suppose the assertion is true for $n$. Then there exists a constant $K_{n}$ such that $\overline{F^{(n)}}(x) \leq n \bar{F}(x)+K_{n} \bar{F}(x)^{2}$ for $x>0$.

Since $F \in \mathscr{S}^{2}$ it follows that

$$
\int_{0-}^{x}\{\bar{F}(x-u)-\bar{F}(x)\} d F(u)=\overline{F^{(2)}}(x)-2 \bar{F}(x)+\bar{F}(x)^{2}=o\left(\bar{F}(x)^{2}\right),
$$

hence

$$
\int_{0-}^{x} \bar{F}(x-u) d F(u)=\bar{F}(x)-\bar{F}(x)^{2}+o\left(\bar{F}(x)^{2}\right)
$$

and

$$
\int_{0-}^{x} \bar{F}(x-u)^{2} d F(u)=\bar{F}(x)^{2}+o\left(\bar{F}(x)^{2}\right)
$$


Hence, for $A \in(0, x)$

$$
\begin{aligned}
\overline{F^{(n+1)}} & (x)=\bar{F}(x)+\int_{0-}^{A} \overline{F^{(n)}}(x-u) d F(u)+\int_{A}^{x} \overline{F^{(n)}}(x-u) d F(u) \\
\leq & \bar{F}(x)+\overline{F^{(n)}}(x-A) F(A)+\int_{A}^{x}\left\{n \bar{F}(x-u)+K_{n} \bar{F}(x-u)^{2}\right\} d F(u) \\
= & \bar{F}(x)+\left\{n \bar{F}(x)-\left(\begin{array}{l}
n \\
2
\end{array}\right) \bar{F}(x)^{2}\right\} F(A)+n \bar{F}(x) F(x)+K_{n} \bar{F}(x)^{2} \\
& -\int_{0-}^{A}\left\{n \bar{F}(x-u)+K_{n} \bar{F}(x-u)^{2}\right\} d F(u)+o(\bar{F}(x))^{2}
\end{aligned}
$$

by Theorem 2. From equation (6) it follows that the last integral equals $\left\{n \bar{F}(x)+K_{n} \bar{F}(x)^{2}\right\} F(A)+o\left(\bar{F}(x)^{2}\right)$. Collecting all terms we find

$$
\varlimsup_{x \rightarrow \infty} \frac{\overline{F^{(n+1)}}(x)-(n+1) \bar{F}(x)}{\bar{F}(x)^{2}} \leq-n-\left(\begin{array}{l}
n \\
2
\end{array}\right) F(A)+K_{n} \bar{F}(A) .
$$

Now take the limit as $A \rightarrow \infty$ on the right-hand side. In view of the left-hand inequality in Lemma 1 the proof is complete.

Our next result shows that assumption (5) in Theorem 1 may be weakened at the cost of an additional integrability condition in order to obtain a sufficient condition for $\mathscr{S}^{2}$. The method of proof is as in Murphree [11].

Theorem 5. Suppose $F$ satisfies (8) and assume there exists a constant $c$ such that

$$
\psi(x)-\psi(u) \leq \exp \left(-\frac{u}{x} \psi(x)+c\right) \quad \text { for } u \in\left[\frac{x}{2}, x\right]
$$

and

$$
\int_{0}^{\infty} \exp \left\{\frac{1}{2}(\psi(2 x)-2 \psi(x))\right\} d \psi(x)<\infty
$$

Then $F \in \mathscr{S}^{2}$.

Proof. From the assumption (16) it follows that $\psi(x)-\psi(x / 2) \rightarrow 0$ as $x \rightarrow \infty$. Hence $\bar{F}$ is slowly varying. Since

$$
\begin{aligned}
\frac{\overline{F^{(2)}}(x)-2 \bar{F}(x)}{\bar{F}(x)^{2}}= & 2 \int_{0-}^{x / 2} \frac{\bar{F}(x-y)-\bar{F}(x)}{\bar{F}(x)^{2}} d F(y) \\
& +\frac{\bar{F}(x / 2)}{\bar{F}(x)}\left(\frac{\bar{F}(x / 2)}{\bar{F}(x)}-2\right)
\end{aligned}
$$

it is sufficient to show that the integral on the right-hand side tends to zero as $x \rightarrow \infty$. Note that $(16)$ implies $\psi(x)-\psi(u) \rightarrow 0(x \rightarrow \infty)$ for $u \in[x / 2, x]$ 
and hence there exists $c_{0}$ such that $\psi(x) \leq \psi(u)+c_{0}$ for $u \in[x / 2, x]$, $x>0$, which implies

$$
\frac{\psi(x)}{x} \leq \frac{\psi(u)}{u}+\frac{c_{0}}{u} \text { for } u \in\left[\frac{x}{2}, x\right], x>0,
$$

and, in general,

$$
\frac{\psi(x)}{x} \leq \frac{\psi(u)}{u}+\frac{2 c_{0}}{u} \text { for } 0<u \leq x .
$$

Since $\psi(x)-\psi(u) \rightarrow 0$ for $u \in[x / 2, x]$, it follows that for $0<y<x / 2$ and $x$ sufficiently large

$$
\begin{aligned}
e^{\psi(x)} & \left\{e^{\psi(x)-\psi(x-y)}-1\right\} e^{-\psi(y)} \leq 2 e^{\psi(x)}\{\psi(x)-\psi(x-y)\} e^{-\psi(y)} \\
& \leq 2 \exp \left\{\frac{y}{x} \psi(x)-\psi(y)+c\right\} \leq 2 \exp \left\{y\left(\frac{\psi(2 y)}{2 y}+\frac{c_{0}}{y}\right)-\psi(y)+c\right\} \\
& \leq 2 e^{c_{0}+c} \exp \left\{\frac{1}{2}(\psi(2 y)-2 \psi(y))\right\} .
\end{aligned}
$$

Since $F$ satisfies $(8)$ we may apply Lebesgue's dominated convergence theorem to the integral on the right-hand side of (18) to find that the limit of the integral is 0 as $x \rightarrow \infty$.

As in the case of the class of subexponential distribution functions, in order to give necessary and sufficient conditions one needs an additional regularity assumption. The following result is an analogue of Pitman's [14] necessary and sufficient condition for the class $\mathscr{S}^{2}$.

THEOREM 6. Suppose $\psi$ has a derivative $\psi^{\prime}$ which is eventually nonincreasing and $\psi^{\prime}(x) \rightarrow 0(x \rightarrow \infty)$. Then $F \in \mathscr{S}^{2}$ if and only if $x \psi^{\prime}(x) \rightarrow$ $0(x \rightarrow \infty)$.

Proof. Let

$$
J:=\frac{\overline{F^{(2)}}(x)-2 \bar{F}(x)}{\bar{F}(x)^{2}}+1=\int_{0}^{x} e^{\psi(x)}\left\{e^{\psi(x)-\psi(x-y)}-1\right\} e^{-\psi(y)} \psi^{\prime}(y) d y .
$$

Suppose first that $F \in \mathscr{S}^{2}$, that is, $J \rightarrow 0$ as $x \rightarrow \infty$. Choose $a$ so that $\psi^{\prime}(x)$ is non-increasing in $[a, \infty)$. Since $\psi$ is non-decreasing we have, if $x \geq a$,

$$
\begin{aligned}
J & \geq \int_{0}^{x}(\psi(x)-\psi(x-y)) \psi^{\prime}(y) d y \\
& \geq \int_{0}^{x-a} \int_{x-y}^{x} \psi^{\prime}(z) d z \psi^{\prime}(y) d y \\
& \geq \psi^{\prime}(x) \int_{0}^{x-a} y \psi^{\prime}(y) d y \geq\left(\psi^{\prime}(x)\right)^{2} \int_{0}^{x-a} y d y,
\end{aligned}
$$

whence $\left(x \psi^{\prime}(x)\right)^{2} \rightarrow 0$. 
Now suppose that $x \psi^{\prime}(x) \rightarrow 0$. It follows that $\bar{F}(x)$ is slowly varying. Split the range of integration of $J$ at $x / 2$ to give two integrals, $J_{1}$ and $J_{2}$. If $y \leq x / 2$ then $x-y \rightarrow \infty$ and using the mean value theorem and that $y \psi^{\prime}(x) \rightarrow 0$ uniformly with respect to $y \leq x / 2$ as $x \rightarrow \infty$, we find

$$
\begin{aligned}
J_{1} & \leq e^{\psi(x)} \int_{0}^{x / 2}\left(e^{y \psi^{\prime}(x / 2)}-1\right) e^{-\psi(y)} \psi^{\prime}(y) d y \\
& \sim \psi^{\prime}(x / 2) e^{\psi(x)} \int_{0}^{x / 2} y e^{-\psi(y)} \psi^{\prime}(y) d y .
\end{aligned}
$$

The integral equals

$$
-\int_{0-}^{x / 2} y d \bar{F}(y)=\int_{0}^{x / 2} \bar{F}(y) d y-(x / 2) \bar{F}(x / 2)=o(x \bar{F}(x / 2)),
$$

because $\bar{F}$ is slowly varying. But $\bar{F}(x)=\exp (-\psi(x)) \sim \bar{F}(x / 2)$ and since $\psi^{\prime}(x)=-\bar{F}^{\prime}(x) / \bar{F}(x), J_{1}=o\left(x \bar{F}^{\prime}(x / 2) / \bar{F}(x / 2)\right) \rightarrow 0$. To deal with $J_{2}$ observe that for any $\varepsilon>0$ we have $\psi^{\prime}(y)<\varepsilon / y$ for all $y$ large enough, and hence

$$
\begin{aligned}
J_{2} & \leq[\varepsilon / \bar{F}(x)] \int_{x / 2}^{x}[(\bar{F}(x-y) / \bar{F}(x))-1] \bar{F}(y) y^{-1} d y \\
& \leq\left[2 \varepsilon \bar{F}(x / 2) / x \bar{F}(x)^{2}\right] \int_{0}^{x / 2} \bar{F}(z) d z \\
& \sim\left[2 \varepsilon \bar{F}(x / 2) / x \bar{F}(x)^{2}\right][x \bar{F}(x / 2) / 2] \rightarrow \varepsilon
\end{aligned}
$$

where we have used the slow variation of $\bar{F}$. It follows that $J \rightarrow 0$, and the theorem follows.

REMARKS. (1) If $F$ satisfies the sufficient condition of Theorem 6 and $G$ is a d.f. on $[0, \infty)$ satisfying (11), then $G \in \mathscr{S}^{2}$.

(2) Although it is not immediately apparent, Theorem 6 is closely related to Pitman's [14, Theorem II]. Under the same supplementary condition as in Theorem 6 he gives a necessary and sufficient condition for membership of $\mathscr{S}$. Let

$$
I(x)=\int_{0}^{x}\left(e^{y \psi^{\prime}(x)}-1\right) e^{-\psi(y)} \psi^{\prime}(y) d y .
$$

Suppose $\psi^{\prime}(x)$ ultimately decreases to zero as $x \rightarrow \infty$. Then Pitman's result is equivalent to the following: $F \in \mathscr{S}$ if and only if $I(x) \rightarrow 0$ as $x \rightarrow \infty$. We can obtain a criterion for membership of $\mathscr{S}^{2}$ by complementing Pitman's condition with a convergence rate requirement:

$$
F \in \mathscr{S}^{2} \text { if and only if } I(x) / \bar{F}(x) \rightarrow 0 \text { as } x \rightarrow \infty .
$$

It is not very difficult to show that this criterion is equivalent to the apparently simpler criterion of Theorem 6 . The proof of this equivalence relies strongly 
on the fact that our criterion ensures that $\bar{F}$ is slowly varying. This additional structure is not available in Pitman's situation and consequently it is not at all obvious that his criterion for membership of $\mathscr{S}$ can be simplified in any useful way.

\section{Examples}

Next we construct an example of a distribution function satisfying (8) but not in $\mathscr{S}^{2}$. Define $x_{0}=0$ and the piecewise linear function $\psi$ as follows: take $x_{n}:=x_{n-1}+n e^{n-1} \quad(n \geq 1)$ and $\psi\left(x_{n}\right)=n \quad(n \geq 0)$. Then $\psi(x)=\int_{0}^{x} \psi^{\prime}(s) d s \quad(x \geq 0)$ with $\psi^{\prime}(x)=n^{-1} e^{-n+1}$ on $\left(x_{n-1}, x_{n}\right]$ for $n=1,2, \ldots, \psi^{\prime}$ is non-increasing, $\psi^{\prime}(x) \rightarrow 0 \quad(x \rightarrow \infty)$. It follows that $x_{n-1} \psi^{\prime}\left(x_{n}\right) \geq(n-1) e^{n-2} \cdot n^{-1} e^{-n+1} \geq(2 e)^{-1}$ for $n \geq 2$, and hence we have

$$
\begin{aligned}
e^{\psi\left(x_{n}\right)} \int_{x_{n-1}}^{x_{n}}\left(e^{y \psi^{\prime}\left(x_{n}\right)}-1\right) e^{-\psi(y)} \psi^{\prime}(y) d y & \geq \psi^{\prime}\left(x_{n}\right) e^{\psi\left(x_{n}\right)} \int_{x_{n-1}}^{x_{n}} y \psi^{\prime}(y) e^{-\psi(y)} d y \\
& \geq x_{n-1} \psi^{\prime}\left(x_{n}\right)\left\{e^{\psi\left(x_{n}\right)-\psi\left(x_{n-1}\right)}-1\right\} \\
& \geq(2 e)^{-1}(e-1)=\left(1-e^{-1}\right) / 2
\end{aligned}
$$

from which it follows that $F \notin \mathscr{S}^{2}$. On the other hand $F$ satisfies (8) since $\psi^{\prime}(x) e^{\psi(x)} \rightarrow 0 \quad(x \rightarrow \infty)$. This follows because $x_{n} \rightarrow \infty \quad(n \rightarrow \infty)$ and for $x \in\left(x_{n-1}, x_{n}\right]$ we have

$$
0 \leq \psi^{\prime}(x) e^{\psi(x)} \leq n^{-1} e^{-n+1+\psi\left(x_{n}\right)}=e / n .
$$

Note that in the above example $\bar{F}$ is not slowly varying because if $\bar{F}$ is slowly varying then, since $\bar{F}^{\prime}=-\psi^{\prime} e^{-\psi}$ is non-increasing, it follows that $x \bar{F}^{\prime}(x) / \bar{F}(x)=x \psi^{\prime}(x) \rightarrow 0(x \rightarrow \infty)$. On the other hand for $x \in$ $\left(x_{n-1}, x_{n}\right]$ and $n \geq 2$ we have $x \psi^{\prime}(x) \geq x_{n-1} \psi^{\prime}\left(x_{n}\right) \geq(2 e)^{-1}$.

We have seen that $F \in \mathscr{S}^{2}$ implies $\bar{F}$ is slowly varying. Our next example shows that the converse implication is not true, that is, we give an example of a d.f. which is slowly varying but not in $\mathscr{S}^{2}$. We have seen that $\bar{F}(x)=$ $(\log x)^{-1}, x \geq e$, is in $\mathscr{S}^{2}$. We claim that $\bar{F}(x)=[\log x]^{-1}, x \geq e$, is not in $\mathscr{S}^{2}$, where [.] denotes the greatest integer function. Indeed

$$
\begin{aligned}
\overline{F^{(2)}}\left(e^{n}\right) & =2 \bar{F}\left(e^{n}\right)-\bar{F}\left(e^{n}\right)^{2}+2 n^{-1}(n-1)^{-1} \sum_{k=2}^{n} k^{-1}(k-1)^{-1}-n^{-1}(n-1)^{-1} \\
& =\frac{3}{n}-\frac{1}{n-1}+\frac{1}{n^{2}},
\end{aligned}
$$


and hence $\overline{F^{(2)}}\left(e^{n}\right)-2 \bar{F}\left(e^{n}\right)=o\left(\bar{F}\left(e^{n}\right)^{2}\right) \quad(n \rightarrow \infty)$. This example also shows that $\mathscr{S}^{2}$ is not closed under tail equivalence without supplementary conditions such as those in Theorem 3. Moreover if we replace lim inf by lim sup in (2) this example shows that this lim sup can be strictly greater than zero even if the lim inf equals zero.

Next we give an application of the above theory. In order to prove the result we need an inequality which is similar to the Kesten inequality for subexponential distribution functions.

LEMMA 2. Suppose $F \in \mathscr{S}^{2}$ and define the sequences $\beta_{n}\left(x_{0}\right), \gamma_{n}\left(x_{0}\right)$ $(n \geq 1)$ by

$$
\beta_{n}\left(x_{0}\right)=\sup _{x \geq x_{0}}\left\{\overline{F^{(n)}}(x)-n \bar{F}(x)\right\} / \bar{F}(x)^{2}
$$

and

$$
\gamma_{n}\left(x_{0}\right)=\inf _{x \geq x_{0}}\left\{\overline{F^{(n)}}(x)-n \bar{F}(x)\right\} / \bar{F}(x)^{2} .
$$

Then for any $\varepsilon>0$ there exist $k_{0}>0$ not depending on $n$ and $x_{0}=x_{0}(\varepsilon)$ such that

$$
-k_{0}(1+\varepsilon)^{n} \leq \gamma_{n}\left(x_{0}\right) \leq \beta_{n}\left(x_{0}\right) \leq k_{0}(1+\varepsilon)^{n} \text { for } n \geq 2 .
$$

Proof. Note that $\beta_{n}\left(x_{0}\right)$ and $\gamma_{n}\left(x_{0}\right)$ are finite for all $x_{0} \geq 0, n \geq 2$, by Theorem 4 . As in the proof of Theorem 4 it follows that for each $\varepsilon>0$ there exists $x_{0}$ such that

$$
\int_{0-}^{x} \bar{F}(x-u)^{2} d F(u) \leq\left(1+\frac{\varepsilon}{2}\right) \bar{F}(x)^{2} \text { for } x>x_{0},
$$

and hence using (6), we have

$$
\begin{aligned}
\int_{0-}^{x} & \overline{F^{(n)}}(x-u) d F(u)=\left(\int_{0-}^{x-x_{0}}+\int_{x-x_{0}}^{x}\right) \overline{F^{(n)}}(x-u) d F(u) \\
& \leq \int_{0-}^{x-x_{0}}\left\{n \bar{F}(x-u)+\beta_{n}\left(x_{0}\right) \bar{F}(x-u)^{2}\right\} d F(u)+\bar{F}\left(x-x_{0}\right)-\bar{F}(x) \\
& \leq n\left\{\overline{F^{(2)}}(x)-\bar{F}(x)\right\}+\beta_{n}\left(x_{0}\right)(1+\varepsilon / 2) \bar{F}(x)^{2}+c^{*} \bar{F}(x)^{2}
\end{aligned}
$$

for $x>x_{0}$, where $c^{*}$ is a constant. It follows that

$$
\begin{aligned}
& \overline{F^{n+1}}(x)-(n+1) \bar{F}(x)=\int_{0-}^{x} \overline{F^{(n)}}(x-u) d F(u)-n \bar{F}(x) \\
& \leq n\left\{\overline{F^{(2)}}(x)-2 \bar{F}(x)\right\}+\left\{\beta_{n}\left(x_{0}\right)(1+\varepsilon / 2)+c^{*}\right\} \bar{F}(x)^{2},
\end{aligned}
$$

and hence

$$
\beta_{n+1}\left(x_{0}\right) \leq c_{0} n+c^{*}+\beta_{n}\left(x_{0}\right)(1+\varepsilon / 2),
$$


where $c_{0}=\sup _{x>0}\left\{\overline{F^{(2)}}(x)-2 \bar{F}(x)\right\} / \bar{F}(x)^{2}<\infty$. Iteration then gives $\beta_{n}\left(x_{0}\right) \leq \delta(n+\varepsilon / 2)^{n}, n \geq 2$, for some constant $\delta$, which implies $\beta_{n}\left(x_{0}\right) \leq$ $k_{0}(1+\varepsilon)^{n}$. The proof of the inequality for $\gamma_{n}\left(x_{0}\right)$ follows similarly.

Now consider an age dependent branching process with lifetime distribution $F \in \mathscr{S}^{2}$. If $M(t)$ is the expected population size at time $t>0$ of the process with one ancestor and a per capita mean number of offspring $m<1$, then it follows that

$$
M(t)=\sum_{k=0}^{\infty} m^{k}\left\{\overline{F^{(k+1)}}(t)-\overline{F^{(k)}}(t)\right\},
$$

and hence

$$
\begin{aligned}
\frac{M(t)}{}-(1-m)^{-1} \bar{F}(t) \\
\bar{F}(t)^{2} \\
=\sum_{k=0}^{\infty} m^{k} \frac{\left\{\overline{F^{(k+1)}}(t)-(k+1) \bar{F}(t)\right\}-\left\{\overline{F^{(k)}}(t)-k \bar{F}(t)\right\}}{\bar{F}(t)^{2}} .
\end{aligned}
$$

In view of Lemma 2 and Theorem 4 we may apply Lebesgue's dominated convergence theorem to find

$$
M(t)=\frac{\bar{F}(t)}{1-m}-\frac{m}{(1-m)^{2}} \bar{F}(t)^{2}+o\left(\bar{F}(t)^{2}\right)(t \rightarrow \infty) .
$$

Observe that $1-M(t)$ is a d.f. and it follows from (20) and Theorem 3 that this d.f. is in $\mathscr{S}^{2}$.

Although it is not obvious at first sight, results like (20) can have a rather narrow scope of appliability in some situations. To illustrate this, consider the limiting waiting time d.f. $Q(t)$ of a stable $M / G / 1$ queue whose service time d.f. is $B(t)$. If $F(t)$ is the idle time d.f. and $m=Q(0)$ is the limiting probability of not waiting for service then it is well-known that (19) holds where $M(t)=\bar{Q}(t) / m$. It follows that $F \in \mathscr{S}^{2}$ implies $Q \in \mathscr{S}^{2}$ and a version of (20) holds for $\bar{Q}(t)$.

Stability of the queueing system requires that $b=\int_{0}^{\infty} t d B(t)<\infty$. Hence $B(t)=b^{-1} \int_{0}^{t}(1-B(u)) d u$ is a d.f. Moreover the idle time distribution tail function $\bar{F}$ is proportional to $\bar{\beta}(t)$ and hence it follows that $F \in \mathscr{S}^{2}$ if and only if $\beta \in \mathscr{S}^{2}$. If so, $\bar{\beta}(t)=\exp (-\psi(t))$ is slowly varying and the monotone density theorem shows that

$$
t \psi^{\prime}(t)=t \bar{B}(t) / \int_{t}^{\infty} \bar{B}(x) d x \rightarrow 0 .
$$

Conversely, if $\bar{B}$ is regularly varying then (21) can be satisfied only if the index is -1 , that is, $\bar{B}(t)=L(t) / t$ where $L$ is slowly varying, and we must 
have $\int_{t}^{\infty} L(x) d x / x<\infty$. For this form of $\bar{B}$, equation (21) is satisfied because Fatou's lemma yields $\int_{1}^{\infty}[L(t u) / L(t]) d u \rightarrow \infty$.

Note that $\psi^{\prime}(t)=L(t) / t \int_{t}^{\infty} L(x) d x / x$, so that if this is eventually nonincreasing we conclude from Theorem 6 that $F \in \mathscr{S}^{2}$. These conditions are obviously satisfied by, for example, $L(t)=K(\log (e+t))^{-1-\delta}$, where $K$ is a normalising constant and $\delta>0$.

Summarising, we see that among service time distributions having a regularly varying upper tail, an approximation for the stable waiting time distribution of the form of $(20)$ is effectively restricted to those service time distributions whose index of variation is -1 .

\section{Acknowledgement}

The authors are indebted to the referee for making the connection with functions of slow variation with remainder.

\section{References}

[1] K. B. Athreya and P. E. Ney, Branching processes, Springer-Verlag (1972).

[2] N. H. Bingham, C. M. Goldie and J. L. Teugels, Regular variation, Cambridge University Press (1987).

[3] V. P. Chistyakov, 'A theorem on sums of independent, positive random variables and its application to branching processes', Theory Prob. and Appl. 9 (1964), 640-648.

[4] D. B. H. Cline, 'Convolution tails, product tails and domains of attraction', Prob. Th. Rel. Fields 72 (1986), 529-557.

[5] P. Embrechts and C. M. Goldie, 'On convolution tails', Stochastic Proc. Appl. 13 (1982), 263-278.

[6] P. Embrechts, 'Subexponential distribution functions and their applications: a review', Proc. Seventh Brasov Conf. on Prob. Theory, M. Iosifescu, VNU Science Press, Utrecht, (1985), 125-136.

[7] J. L. Geluk, 'On the convolution of functions which belong to a subclass of $L^{1}(0, \infty)$ ', Applicable Analysis 20 (1985), 79-88.

[8] J. L. Geluk and L. de Haan, Regular variation, extensions and Tauberian theorems, CWI tract 40, Centre for Mathematics and Computer Science, Amsterdam (1987).

[9] C. M. Goldie and R. L. Smith, 'Slow variation with remainder: theory and applications', Quart. J. Math. Oxford (2), 38 (1987), 45-71.

[10] W. A. J. Luxemburg, 'On an asymptotic problem concerning the Laplace transform', Applicable Analysis 8 (1978), 61-70.

[11] E. S. Murphree, 'Some new results on the subexponential class', J. Appl. Prob. 26 (1989), 892-897.

[12] E. Omey, 'Asymptotic properties of convolution products of functions', Publ. Inst. Math. 43 (1988), 41-57. 
[13] A. G. Pakes, 'On the tails of waiting-time distributions', J. Appl. Prob. 12 (1975), 555564.

[14] E. J. G. Pitman, 'Subexponential distribution functions', J. Austral. Math. Soc. Ser. A 29 (1980), 337-347.

[15] J. L. Teugels, 'The class of subexponential distributions', Ann. Prob. 3 (1975), 10001011.

[16] E. Willekens, Hogere orde theorie voor subexponentiele verdelingen, Ph.D. thesis, Univ. of Louvain (1986).

Erasmus University

Econometric Institute

P.O. Box 1738

3000 DR Rotterdam

The Netherlands
University of Western Australia

Nedlands, WA 6009

Australia 